Document downloaded from:

http://hdl.handle.net/10251/80122

This paper must be cited as:

Mollar García, MA.; Cembrero Cil, J.; Marí, B. (2016). Photoluminescent properties of electrochemically synthetized ZnO nanotubes. Materials Characterization. 119:152-158. doi:10.1016/j.matchar.2016.07.022.

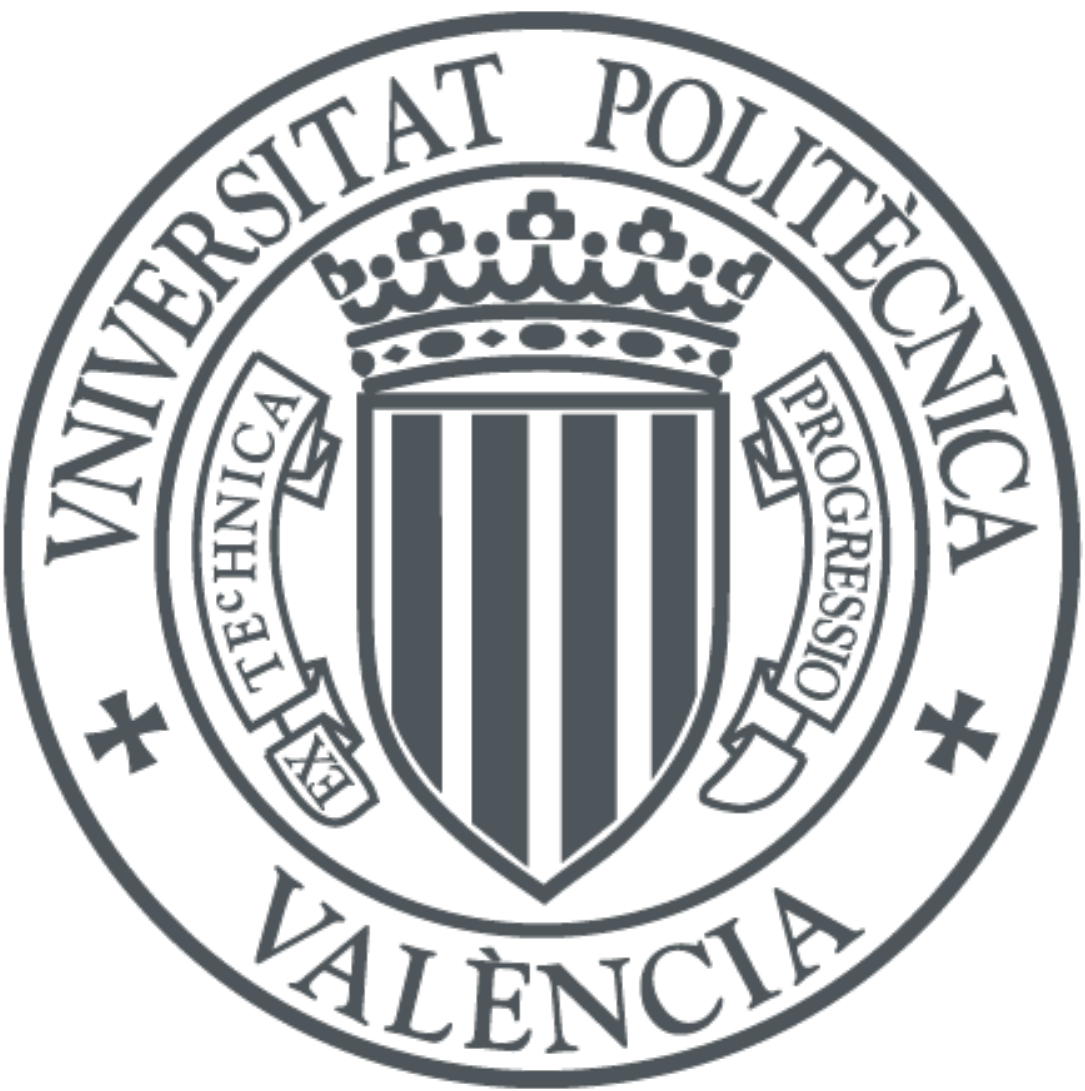

The final publication is available at

http://dx.doi.org/10.1016/j.matchar.2016.07.022

Copyright Elsevier

Additional Information 


\title{
Photoluminescent properties of electrochemically synthetized ZnO nanotubes
}

\author{
J. M. Gracia Jiménez ${ }^{\mathrm{a}}$, J. Cembrero ${ }^{\mathrm{b}}$, M. Mollar ${ }^{\mathrm{c}}$, B. Marí ${ }^{\mathrm{c}},{ }^{*}$ \\ a Instituto de Física, Benemérita Universidad Autónoma de Puebla, Apartado Postal J-48, Puebla, Pue. \\ 72570 México \\ ${ }^{b}$ Departament d'Enginyeria Mecànica i Materials, Universitat Politècnica de Valencia, Camí de Vera \\ s/n, 46071 - València, Spain \\ ${ }^{c}$ Departament de Física Aplicada-IDF, Universitat Politècnica de Valencia, Camí de Vera s/n, 46071 - \\ València, Spain
}

*Corresponding author: bmari@fis.upv.es

\begin{abstract}
$\mathrm{ZnO}$ nanotubes were prepared by a sequential combination of electrochemical deposition, chemical attack and regeneration. $\mathrm{ZnO}$ nanocolumns were initially electrodeposited on conductive substrates and then converted into nanotubes by a process involving chemical etching and subsequent regrowth. The morphology of these $\mathrm{ZnO}$ nanocolumns and derived nanotubes was monitored by Scanning Electron Microscopy and their optical properties was studied by photoluminescence spectroscopy. Photoluminescence were measured as a function of temperature, from 6 to $300 \mathrm{~K}$, for both nanocolumns and nanotubes. In order to study the behaviour of induced intrinsic defect all $\mathrm{ZnO}$ films were annealed in air at $400{ }^{\circ} \mathrm{C}$ and their photoluminescent properties were also registered before and after annealing. The behaviour of photoluminescence is explained taking into account the contribution of different point defects. A band energy diagram related to intrinsic defects was proposed to describe the behaviour of photoluminescence spectra.
\end{abstract}

Keywords: Nanocolumns; Nanotubes; Growth from solution; Zinc Oxide; Defects; Photoluminescence. 


\section{Introduction}

Zinc Oxide $(\mathrm{ZnO})$ is a wide gap semiconductor well known for various potential applications in low-voltage and short-wavelength optoelectronic devices, such as light emitting and laser diodes, optical windows and solar cells [1-3]. In the last few years, owing to its potential applications in ultraviolet optoelectronic devices, a growing number of research projects have been developed using $\mathrm{ZnO}$ as basic material. Nanostructured samples of $\mathrm{ZnO}$ have been prepared by many different techniques, such as plasma-assisted molecular beam epitaxy [4], chemical vapor deposition [5], and pulsed laser deposition [6]. As another approach, electrodeposition (ED) presents several advantages over the above mentioned techniques such as low temperature processing and low cost $[7,8]$. This technique allows controlling nanostructure dimensions by means of the growth parameters [9].

Unintentionally doped $\mathrm{ZnO}$ is always is an n-type semiconductor due to the existence of defects involving $\mathrm{O}$ vacancies $\left(\mathrm{V}_{\mathrm{O}}\right)$ or $\mathrm{Zn}$ interstitial $\left(\mathrm{Zn}_{\mathrm{i}}\right)$, which act as donors. Theoretical calculations based on ab-initio methods support this experimental fact [a]. Owing to these defect-related donors, p-type doping is still a challenge and numerous efforts addressed to reduce this type of native defects have been carried out in the last decade. Indeed, a better understanding of their properties could pave the way for improving the control over optical and electrical properties of $\mathrm{ZnO}$. Recently, a big effort has been made to reduce such defects in order to improve their properties, and several thermal annealing procedures were explored aimed to correct the deficiency of $\mathrm{O}$ and / or modify other native defects [11-13]. Furthermore, it was found that chemical films etching modify the surface morphology of $\mathrm{ZnO}$ [9], and thus some mechanism being sensitive to emission surface quality (e.g. excitons) is also expected to modify its radiative emission.

One approach deals with the exploration of annealing in air to correct the Oxygen deficiency or to modify the nature of other types of native defects. On the other hand, it has been observed that when $\mathrm{ZnO}$ films are exposed to a chemical attack, the surface morphology changes and subsequently the 
emission mechanisms (involving excitons or deep defects), which are sensitive to surface quality, are expected to modify their radiative emission properties [14].

The low temperature synthesis of electrodeposited $\mathrm{ZnO}$ usually requires post annealing treatments in order to enhance optical properties of thin films and reducing native centers thus improving crystallinity [15]. Following the evolution and optimization of optical properties, several studies have been published using reducing and/or oxidizing atmospheres [16-17]. However, the interpretation of these results is controversial regarding the contribution of different defects to the photoluminescence spectra [18-20]. On the other hand, the low temperature electrodeposition of $\mathrm{ZnO}$ makes this technique compatible with the use of polymers for making electroluminescent hybrid devices by means of heterojunctions formed by nanocolumns of electrodeposited $\mathrm{ZnO}$ and a polymer as recently demonstrated by Könenkamp [21].

Photoluminescence (PL) is a non-destructive technique, which directly probes optically active centers that affect the response of $\mathrm{ZnO}$ thin films and their evolution with the specific treatment applied to them. In this experiment, a group of samples was synthesized under the same growing conditions with two objectives: to investigate how atmosphere and annealing temperature could improve optical properties. As the size and surface-volume ratios of the $\mathrm{ZnO}$ columns depend mainly on the growth parameters, such as current density, deposition rate and deposition bath temperature and they hardly influence the emission properties $[9,17]$, special attention was paid to obtain samples with identical morphology.

The aim of this paper is to investigate the PL properties of nanostructured $\mathrm{ZnO}$ films related to their morphology (nanocolumns or nanotubes) and subsequent thermal annealing. The analysis of the PL properties will be useful to shed some light on the structure of native defects in $\mathrm{ZnO}$. A model based on 
the energy band diagram related to deep defects in $\mathrm{ZnO}$ is proposed to understand the PL behaviour of such $\mathrm{ZnO}$ nanostructures.

\section{Experimental}

Nanocolumnar $\mathrm{ZnO}$ films were prepared by electrodeposition (ED) on glass substrates covered with a conductive layer of tin oxide doped with fluorine (FTO). The electrolyte used is a solution of $\mathrm{ZnCl}_{2}\left(5 \times 10^{-3} \mathrm{M}\right)$ and $0.1 \mathrm{M}$ of $\mathrm{KCl}$ in demineralized water $(\rho>16 \mathrm{M} \Omega \cdot \mathrm{cm})$ as used in previous works $[9,15]$. During the ED stages, the solution was saturated with $\mathrm{O}_{2}$ by continuous bubbling. Temperature was controlled with a thermal blanket and remained within $\pm 1^{\circ} \mathrm{C}$. No stirring was applied. A threeelectrode cell configuration was used, $\mathrm{KCl}$ saturated $\mathrm{Ag} / \mathrm{AgCl}$ as reference electrode and a $\mathrm{Pt}$ wire as counter electrode. Measured $\mathrm{pH}$ in the fresh electrolyte was 6.5 . The potential was fixed at $-0.85 \mathrm{~V}$ with respect to the reference electrode. Previous results show that temperature, current density and time exposure help the formation of column growth [8].

The procedure developed to fabricate hollow $\mathrm{ZnO}$ nanocolumns consisted of three steps: (1) column formation by electrodeposition (ED), (2) a chemical attack to produce the hollow structures and eventually (3) regeneration of the hollow nanocolumns again by ED. This last step was required just in case the second step was too aggressive giving hollow nanocolumns but very irregular in shape. Details of the procedure to deposit $\mathrm{ZnO}$ nanotubes can be found elsewhere [22]. After growth of both, $\mathrm{ZnO}$ nanocolumns and nanotubes samples were further annealed in air at $400^{\circ} \mathrm{C}$.

Photoluminescence spectroscopy was performed by excitation with the $325 \mathrm{~nm}$ line of a $\mathrm{He}-\mathrm{Cd}$ laser with an optical power of $30 \mathrm{~mW}$. The setup consisted of a Jovin Yvon TRIAX monochromator coupled to a Si-CCD detector optimized for UV-VIS range. PL measurements were carried out between $6 \mathrm{~K}$ and room temperature. At cryogenic temperature, samples were mounted on the cold finger of a He closed cycle cryostat refrigerator and cooled down to the desired temperature. 


\section{Results and discussion}

\section{Morphology}

Figure 1 shows SEM micrographs of two $\mathrm{ZnO}$ samples under different conditions: a) as grown nanocolums, b) nanotubes created after chemical etching and further regrowth. Observation by means of SEM shows this deposit in form of hexagonal nanocolumns of approximately $100 \mathrm{~nm}$ of base and 500 $\mathrm{nm}$ high [18], partially oriented is the 002 direction of a hexagonal wurtzite structure as inferred form XRD spectra $[8,9]$. Nanotubes obtained after chemical etching and subsequent electrochemical regrowth are identical in form, base and height. The difference consists in the fact that the inner part of the nanocolumns is no longer present after the described process. The walls of the nanotubes are about 20 $\mathrm{nm}$ thick and quite regular along the deposit. Figure $1 \mathrm{c}$ ) shows a detail of the $\mathrm{ZnO}$ nanotubes.

In both morphologies, nanocolumns and nanotubes, no relevant differences were appreciated in the morphology between as-grown and annealed samples.

\section{Photoluminescence}

Figures $2 \mathrm{a}$ and $2 \mathrm{~b}$ show the PL spectra registered at 6,80 and $300 \mathrm{~K}$ of as-grown $\mathrm{ZnO}$ nanocolumns and nanotubes, respectively. As can be seen, both samples show two bands, one in the region of the shallow impurities and excitons (located at 377 and $381 \mathrm{~nm}$, respectively) and one related to deeper levels with two bands centered at 610 and $622 \mathrm{~nm}$, respectively.

It is known that a typical spectrum of $\mathrm{ZnO}$ films has two characteristic peaks: a UV near-bandedge (NBE) whose peak emission is around $377 \mathrm{~nm}[19,22,23]$, and a deep-level (DL) emission, also known as green-band or red-band depending on if they are centred around 500 or $600 \mathrm{~nm}$. A sharp NBE emission peak results from excitons and its position and structure is an indication of crystal quality. The green band is related to transitions involving defects and several point defects, such as oxygen vacancy 
$\left(\mathrm{V}_{\mathrm{O}}\right)$, zinc vacancy $\left(\mathrm{V}_{\mathrm{Zn}}\right)$, interstitial zinc $\left(\mathrm{Zn}_{\mathrm{i}}\right)$, interstitial oxygen $\left(\mathrm{O}_{\mathrm{i}}\right)$ and antisite oxygen $\left(\mathrm{O}_{\mathrm{Zn}}\right)[22,23]$, have been proposed as responsible for this emission in $\mathrm{ZnO}$.

The rise of intensity and shift are the result of a higher concentration of defects and associated impurities related to chemical etching and subsequent regrowth. These actions generate defects located at lower energy, i.e. etching produces defects causing a deterioration of the surface and bulk quality of $\mathrm{ZnO}$. Then etching, not only changes the surface morphology of the films [22], but also increases the rate of defects or impurities existing in the material generating preferentially the ones that shift PL bands towards the red. Moreover, when the temperature increases, the emission reflects the expected behaviour, i.e. the reduction of PL intensity is due to the presence of other non-radiative processes such as electronphonon interactions, which compete with radiative recombination processes.

When both kind of films, nanocolumns and nanotubes, are annealed in air (Figs. 3 a, b) significant changes are observed in both NBE and DL regions. As already observed in the case of chemical etching, a redistribution of impurities and defect levels can be observed in both samples, which causes the dominant peak at low energy to vanish and two new lines appear. These are located at $511 \mathrm{~nm}$ for the nanocolumns and 512 and $642 \mathrm{~nm}$ for the nanotube samples, respectively. Most notably, in this region, the intensity of the new lines in the etched sample (nanotubes) is almost the same, unlike in as-grown nanocolumns and nanotubes (Fig. 2). In addition, in the nanocolumnar sample, the lowest energy PL band appears as a shoulder that is superimposed to that of $511 \mathrm{~nm}$ and does not form a defined band. In the NBE region, the bands of both films drastically reduce their average FWHM, and it is observed that this is the result of the superposition of several lines, some appearing as peaks and a few others as shoulders.

Thus, as seen, thermal treatment modifies the distribution and concentration of defects and impurities, dissociating some and allowing others. When nanocolumns are annealed, the broad band centred at $610 \mathrm{~nm}$ (Fig. 2a) narrows and shifts to lower wavelength (511 nm) (Fig. 3a). Noteworthy, the 
intensity of this DL emission for annealed nanocolumns increases about one order of magnitude. Besides a shoulder at higher wavelengths is also observed. In the case of annealed nanotubes the broad band located at $622 \mathrm{~nm}$ (Fig. 2b) splits into two new bands with similar intensities and located at 512 and 642 nm, respectively (Fig. 3b).

Impurities or defects involved in these bands have not been fully identified yet. However, the defects and impurities associated with the first PL band dissociate or reduce their concentration allowing the appearance of the others. It is likely that type of defects or impurities which originate the $622 \mathrm{~nm}$ band consist of one or two levels associated with impurities or defects such as $\mathrm{Zn}$ vacancies [Vzn], O vacancies $\left[\mathrm{V}_{\mathrm{O}}\right]$ or $\mathrm{Zn}$ interstitials $\left[\mathrm{Zn}_{\mathrm{i}}\right]$ and oxygen interstitials $\left[\mathrm{O}_{\mathrm{i}}\right]$ [2-4], or defects composed of two complexes such as $\left[\mathrm{V}_{\mathrm{O}}-\mathrm{Zn}_{\mathrm{i}}\right]$ [5]. As annealing in air causes the incorporation of oxygen to the material, the concentration of $\mathrm{V}_{O}$ and complexes involving $\mathrm{V}_{O}$ must be reduced. This will increase the concentration of at least isolated $\mathrm{Zn}$, which is reflected in the appearance of the band at $512 \mathrm{~nm}$, which has been associated with a donor $\left(\mathrm{Zn}_{\mathrm{i}}\right)$ - acceptor $(\mathrm{Vzn})$ transition. As a preliminary conclusion it can be said that annealing improves the quality of the surface and reduces the defects and impurities in the films.

To better show the similarities and differences between PL spectra of $\mathrm{ZnO}$ nanocolumns and nanotubes before and after annealing, Figures 4 were plotted to compare PL spectra recorded at $6 \mathrm{~K}$. Figure 4a shows the NBE region, which as mentioned involved excitons and shallow impurities. Figure $4 \mathrm{~b}$ shows the DL region, where defects with deeper energies in the bandgap are involved. For as-grown nanocolumnar samples a vast rise of PL intensity is observed in the NBE region, which is comparable to that observed in the DL region and is higher than observed for as grown nanotubes. Furthermore, both samples, as grown nanocolumns and nanotubes (Fig 4a) show a similar shape, which comes from the superposition of several PL bands. The dominant peak of these PL spectra is located at $371 \mathrm{~nm}$ and the rest appears as shoulders at 370, 373 and $381 \mathrm{~nm}$, while for annealed samples the main peaks are at 370 and $381 \mathrm{~nm}$ with a shoulder at $375 \mathrm{~nm}$. This is mainly due to the annealing that reduces defects and 
improves the surface quality of the samples. In these conditions excitons and recombination involving shallow defects effectively compete with other emission mechanisms.

Concerning the DL region in both cases, a split of the initial PL (located at 620-622 nm) is observed before any thermal annealing. This red-band splits into two news bands, one located at $511 \mathrm{~nm}$, which can be considered a green-band and a second red-band centred at $642 \mathrm{~nm}$. The difference between nanocolumns and nanotubes is that the red-band related to annealing is more intense in the case of nanotubes than for nanocolumns. This result could be expected from the shape of the red-bands in the as-grown samples because the red-band for $\mathrm{Zn}$ nanotubes is more intense and is more red-shifted (622 $\mathrm{nm})$ than the red-band in $\mathrm{ZnO}$ nanocolumns $(610 \mathrm{~nm})$.

As mentioned above the intensity of the NBE line depends on crystal quality allowing the formation of excitons but also on other competitor mechanisms such as transitions through the DL band or through other non-radiative levels. Hence an increase of the exciton emission intensity reveals an exhaustion of the alternative recombination paths [24].

To shed light on the effect of the chemical attack, regeneration and thermal annealing a fitting procedure for all PL spectra were carried out. In the DL region the PL peaks were deconvoluted for all studied samples. Figures $5 \mathrm{a}, \mathrm{b}, \mathrm{c}, \mathrm{d}$ show the different components for PL spectra recorded at $6 \mathrm{~K}$ for all the studied samples: a) nanocolumns, b) nanotubes, c) annealed nanocolumns and d) annealed nanotubes. As can be seen, the PL spectra for the as-grown nanocolumns and nanotubes consist of two overlapping emission bands centred at 583 and $656 \mathrm{~nm}$ for as-grown nanocolumns and 591 and 667 for regenerated nanotubes. The emission bands centred at 583 and $591 \mathrm{~nm}$ belong to the region known as yellow emission for $\mathrm{ZnO}$ [25], while bands centred at 656 and $667 \mathrm{~nm}$ are usually labelled as red emission [26].

The chemical etching and subsequent regeneration performed in the synthesis of nanotubes produce a red shift of about $10 \mathrm{~nm}$ and a narrowing of the FWHM of about $2 \mathrm{~nm}$. Furthermore, the intensity of its PL bands increases about 8 times with respect to the as-grown nanocolumns (Fig. 5a and 
5b). The change in the position and FWHM of PL peaks indicates a redistribution of defects, while the increase in the emission intensity is related to an increase in the density of defects. These defects are likely to be associated to the incorporation of $\mathrm{O}$ and $\mathrm{Zn}$ during the regeneration process.

On the other hand, it is generally accepted that the green emission stems from the recombination of one electron in an oxygen vacancy $\left(\mathrm{V}_{\mathrm{O}}\right)$ with a hole coming from the valence band and the red emission is the result of the recombination of an electron in a $V_{O}$ with a hole coming from a zinc vacancy $\left(V_{Z n}\right)$. As a result, the red shift and narrowing of PL bands should correspond to these mechanisms. That means that both emission bands are associated to the same defect, Vo. Furthermore, the chemical etching modifies the surface sample until transforming nanocolumns into nanotubes and creating a high density of $\mathrm{O}$ and $\mathrm{Zn}$ vacancies near the internal surface of the nanotube. The larger number of these defects in nanotubes than in nanocolumns would explain the increase in the intensity of PL bands in the regenerated nanotubes, meaning that the regeneration does not completely recover the defects produced during the chemical etching. Therefore, the mechanisms for this radiative recombination can be assigned unambiguously to the transitions of $\mathrm{V}_{\mathrm{O}}-\mathrm{VB}$ and $\mathrm{V}_{\mathrm{O}}-\mathrm{V}_{\mathrm{Zn}}$. Then, the chemical etching and subsequent regeneration do not only modify the morphology of the nanocolumns but also modify the emission due to the proliferation and redistribution of vacancy related defects.

Figures 5 c) and d) display the deconvolution of the DL band for the nanocolumns and nanotubes annealed in air at $400{ }^{\circ} \mathrm{C}$ for one hour. As can be observed, in both samples the thermal annealing redistributes and increases the impurity levels in such a way that the DL bands are now the superposition of three bands. It is noteworthy to note that PL peaks in the DL region are shifted to shorter wavelengths and FWHMs are narrower for nanotubes than for nanocolumns (see Table I).

After thermal annealing, the emission of nanocolumns increases, becoming similar in intensity the emission of nanotubes. Taking into account that the sole morphological difference between both samples resides in the higher area for nanotubes than for nanocolumns, the incorporation of $\mathrm{O}$ during the 
annealing should be more efficient for nanotubes, resulting in a reduction of $\mathrm{V}_{O}$. This effect explains that red and green emission bands associated to Vo have narrower a FWHM and are blue-shifted with respect to nanocolumns. In addition, the difference between red bands is the result of morphological differences as in nanotubes there should be a larger amount of $\mathrm{VZn}$ than in nanocolumns, which remain practically unaltered with the annealing. Taking into account that the main difference between the two groups of samples, nanocolumns and nanotubes, is the higher surface in nanotubes due to the existence of both inner and outer faces than in nanocolumns, the incorporation of oxygen during the annealing should be more efficient, resulting in a decrease of $\mathrm{V}_{O}$. The decrease of the amount of $\mathrm{V}_{\mathrm{O}}$ produces a blue shift of the position and a narrowing of the FWHM with respect to that observed in nanocolumns. Moreover, the difference in intensity between the red lines is also the result of the morphological differences, since in nanotubes there should be a greater concentration of $\mathrm{V}_{\mathrm{Zn}}$ that in nanocolumns, which remain unchanged by the annealing.

Further, the annealing generates a third line (DL3) located at lower wavelength, $496 \mathrm{~nm}$ for nanocolumns and $488 \mathrm{~nm}$ for nanotubes. As for the yellow and red lines the blue line has narrower FWHM and again is located at shorter wavelength when emitted from nanotubes than when emitted from nanocolumns. This shift to higher energies is likely due to the reduction in size in the walls of nanotubes, which have a thickness of about $20 \mathrm{~nm}$. The emission line located at $496 \mathrm{~nm}$ has been previously reported $[3,27]$ in Ag-doped $\mathrm{ZnO}$ samples, however to our knowledge the $488 \mathrm{~nm}$ emission line has not yet been reported. We believe that the $488 \mathrm{~nm}$ blue emission line should be due to the nanometric size of the walls of nanotubes.

Figure 7 shows a band diagram schema of the energy levels of involved in the photoluminescence emission of nanotubes and nanocolumns before and after thermal annealing. All radiative transitions in the called DL region have been summarized in the schema. Chemical etching and subsequent regrowth results in a shift to longer wavelengths (shorter energies). After thermal annealing additional peaks 
appear, being the trend to shift to shorter wavelengths. Similar results for have been reported elsewhere $[12,13]$.

Furthermore, the envelope of the bands in the region NBE is formed by two lines (NBE1 and NBE2) for films independently of any chemical etching and regrowth. PL lines corresponding to the samples with etching and regeneration have energy peaks at lower energies and slightly increase in intensity and FWHM (See Table I). As in the case of deep impurity region, changes obey to redistribution and increase of energy levels associated with defects and impurities generated by the etching and regeneration in this region. Considering the average FWHM of the lines (Table I), these lines cannot be associated to excitons, because they have higher values than those reported for excitons. So its origin is associated with impurity-band transitions (FB or BF) or with donor-acceptor pair (DAP), where the levels involved should correspond to shallow defect levels.

When thermal annealing treatment is applied to nanocolumns and nanotubes films, the impurity levels or defects reduce its amount and redistribute because after deconvolution five lines are obtained. The position and FWHM of such PL lines (Table I) can be associated to transitions involving the bound exciton (NBE3 and NBE4), and the free exciton plus one or two phonon replicas (NBE5, NBE6). The zero phonon line is not observable because of the filter used. The highlight of these results is that in general PL lines emitted from nanotubes have narrower FWHM than those of columns. It is known that for excitons to be observed there should be a low concentration of impurities capable to trap them and good surface quality, which is reflected in their FWHM of this emission lines. Then it can be concluded that the thermal annealing has a greater influence on the surface quality of nanotubes compared to that of the nanocolumns.

\section{Conclusion}

$\mathrm{ZnO}$ nanotubes were prepared by a sequential combination of electrochemical deposition, chemical 
attack and regeneration of $\mathrm{ZnO}$ nanocolumns deposited on polycrystalline conductive FTO covered glass. $\mathrm{ZnO}$ nanocolumns were initially electrodeposited on conductive substrates and then converted into nanotubes. The morphology of these $\mathrm{ZnO}$ nanocolumns and subsequent nanotubes were monitored by SEM and their optical properties were studied by photoluminescence spectroscopy. Photoluminescence were measured as a function of temperature, from 6 to $300 \mathrm{~K}$, for both nanocolumns and nanotubes. In order to study the behaviour of induced intrinsic defect all $\mathrm{ZnO}$ films were annealed in air at $400{ }^{\circ} \mathrm{C}$ and their photoluminescent properties were also registered before and after annealing. The behaviour of photoluminescence is explained taking into account the contribution of different point defects. A band energy diagram related to intrinsic defects was proposed to describe the behaviour of photoluminescence. These kind of hollow nanocolumns provide a suitable pattern with very high effective surfaces to develop devices based in one-dimensional (1D) nanostructures that can be coated or filled with other thin layers, nanoparticles or organic molecules.

\section{Acknowledgments}

This work was supported by Ministerio de Economía y Competitividad (ENE2013-46624-C4-4-R) and Generalitat valenciana (Prometeus 2014/044). 


\section{References}

[1] M.H. Huang, S. Mao, H. Feick, H. Yan, Y.Y. Wu, H. Kind et al. Room-temperature ultraviolet nanowire nanolasers. Science 2010; 292:1897-9.

[2] L. Grigorjeva, D. Millers, J. Grabis, C. Monty, A. Kalinko, K. Smits et al. Luminescence Properties of ZnO Nanocrystals and Ceramics. IEEE Transactions on nuclear science 2008;55:1551-5.

[3] R. T. Sapkal, S. S. Shinde, A. R. Babar, A. V. Moholkar, K. Y. Rajpure, C. H. Bhosale. Preparation, structural and optical properties of nanocrystalline $\mathrm{ZnO}$ doped with luminescent Ag-nanoclusters, Mater. Express. 2012;2:64-70.

[4] Y. Chen, H. Ko, S. Hong, T. Yao. Layer-by-layer growth of $\mathrm{ZnO}$ epilayer on $\mathrm{Al}_{2} \mathrm{O}_{3}(0001)$ by using a MgO buffer layer. Appl. Phys. Lett. 2000;76:559-61.

[5] D.C. Reynolds, D.C. Look, B. Jogai. Optically pumped ultraviolet lasing from ZnO. Solid State Commun. 1999;99:873-5.

[6] Y.R. Ryu, S. Zhu, S.W. Han, H.W. White, P.F. Miceli, H.R. Chandrasekhar. ZnSe and ZnO film growth by pulsed-laser deposition. Appl. Surf. Sci. 1998;127:496-9.

[7] S. Peulon, D. Lincot. Cathodic electrodeposition from aqueous solution of dense or open-structured zinc oxide films. Adv. Mater. 1996;8:166-70.

[8] J. Cembrero, M. Perales, M. Mollar, B. Marí. Obtención de columnas de ZnO. Variables a controlar

(I) Bol. Soc. Es. Ceram. V. 2003;42:379-87.

[9] J. Cembrero, A. Elmanouni, B. Hartiti, M. Mollar, B. Marí. Nanocolumnar ZnO films for photovoltaic applications. Thin Solid Films 2004;451-452:198-202.

[10] X. Teng, H. Fan, S. Pan, C. Ye, G. Li. Abnormal photoluminescence of ZnO thin films on ITO glass. Mater. Lett. 2007;61:201-4. 
[11] D. Kim, G. Lee, Y. Kim. Interaction of zinc interstitial with oxygen vacancy in zinc oxide: An origin of n-type doping. Solid State Commun. 2012;52:1711-4.

[12] V. Kumar, V. Kumar, S. Som, A. Yousif, N. Singh, O.O. Nwaeaborwa, A. Kapoor, H.C. Swart. Effect of Effect of annealing on the structural, morphological and photoluminescence properties of $\mathrm{ZnO}$ thin films prepared by spin coating. J. Colloid. Interface Sci. 2014;428:8-15.

[13] J. Han, P.Q. Mantas, A.M.R. Senos. Defect chemistry and electrical characteristics of undoped and Mn-doped ZnO. J. Eur. Ceram. Soc. 2002;22:49-59.

[14] J.R. Sadaf, M.Q. Israr, S. Kishwar, O.Nur, M. Willander. White Electroluminescence Using ZnO Nanotubes/GaN Heterostructure Light-Emitting Diode. Nanoscale Res. Lett. 2010;5-957-60.

[15] B. Marí, M. Mollar, A. Mechkour, B. Hartiti, M. Perales, J. Cembrero. Optical properties of nanocolumnar ZnO crystals. Microelectron. J. 2004;35:79-82.

[16] B. Marí, F.J. Manjón, M. Mollar, J. Cembrero, R. Gómez. Photoluminescence of thermal-annealed nanocolumnar $\mathrm{ZnO}$ thin films grown by electrodeposition. Appl. Surf. Sci. 2006;252:2826-31.

[17] B. Marí, J. Cembrero, F.J. Manjón, M. Mollar, R. Gómez. Raman Measurements on nanocolumnar ZnO Crystals. Phys. Stat. Solidi (a) 2005;202:1602-5.

[18] Q. Wang, G. Wang, J. Jie, X. Han, B. Xu, J.G. Hou. Annealing effect on optical properties of ZnO films fabricated by cathodic electrodeposition. Thin Solid Films 2005;492:61-65.

[19] B. Lin, Z. Fu, Y. Jia. Green luminescence center in undoped zinc oxide films deposited on silicon substrates. Appl. Phys. Lett. 2001;79:943.5.

[20] F. Leiter, H. Alves, D. Pfisterer, N.G. Romano, D.M. Hofmann, B.K. Meyer. Oxygen vacancies in. ZnO. Physica B 2003;201:340-342.

[21] R. Könenkamp, R.C. Word, M. Godinez. Ultraviolet electroluminescence from ZnO/polymer heterojunction light-emitting diodes. Nano Letters 2005;5: 2005-8.

[22] J. Cembrero, D. Busquets-Mataix, E. Rayón, M. Pascual, M. A. Pérez Puig, B. Marí. Control parameters on the fabrication of ZnO hollow nanocolumns. Mat. Sci. Semicon. Proc. 2013;16:211-6. 
[23] J. Lim, K. Shin, H.W. Kim, C. Lee. Effect of annealing on the photoluminescence characteristics of ZnO thin films grown on the sapphire substrate by atomic layer epitaxy. Mat. Sci. Eng. B 2004;107: 3014.

[24] J.A. Sans, A. Segura, M. Mollar, B. Marí. Optical properties of thin films of ZnO prepared by pulsed laser deposition. Thin Solid Films 2004;453:251-5.

[25] H. Morkoç, U. Özgür, Zinc Oxide. Fundamentals, Materials and Device Technology. Wiley-VCH; 2009.

[26] Bin Zhao, Ming-Ming Jiang, Dong-Xu Zhao, Yang Li, Fei Wang, De-Zhen Shen. Electrically driven plasmon mediated energy transfer between $\mathrm{ZnO}$ microwires and $\mathrm{Au}$ nanoparticles. Nanoscale $2015 ; 7: 1081-9$.

[27] M. A. Myers, V. Khranovsky, J. Jian, J. H. Lee, H. Wang, H. Wang. Photoluminescence study of ptype versus n-type Ag-doped ZnO films. J. Appl. Phys. 2015;118:065702. 


\section{Figure captions}

Figure 1. SEM images of electrodeposited $\mathrm{ZnO}$ nanostructures: a) As deposited $\mathrm{ZnO}$ nanocolumns, b) $\mathrm{ZnO}$ nanotubes after etching and regrowth, c) detail of nanotubes showing the thickness of the wall.

Figure 2. Photoluminescence spectra of as grown electrodeposited $\mathrm{ZnO}$ nanostructures: a) As deposited $\mathrm{ZnO}$ nanocolumns, b) $\mathrm{ZnO}$ nanotubes after etching and regrowth of $\mathrm{ZnO}$ nanocolumns.

Figure 3. Photoluminescence spectra of electrodeposited $\mathrm{ZnO}$ nanostructures after thermal annealing: a) Nanocolumns, b) Nanotubes.

Figure 4. Comparison of photoluminescence spectra recorded at $6 \mathrm{~K}$ before and after annealing for $\mathrm{ZnO}$ nanostructures: a) Excitonic part (NBE region), b) Deep levels (DL region)

Figure 5. Fit of PL spectra in the DL region recorded at $6 \mathrm{~K}$ for all samples studied in this work. a) Nanocolumns, b) Nanotubes, c) annealed nanocolumns, d) annealed nanotubes.

Figure 6. Fit of PL spectra in the NBE region recorded at $6 \mathrm{~K}$ for the annealed nanotubes.

Figure 7. Band diagram schema to model the energy levels involved defects in radiative transition in the called DL region. CE and TA stand for Chemical Etching and Thermal Annealing, respectively. 


\section{FIGURES}

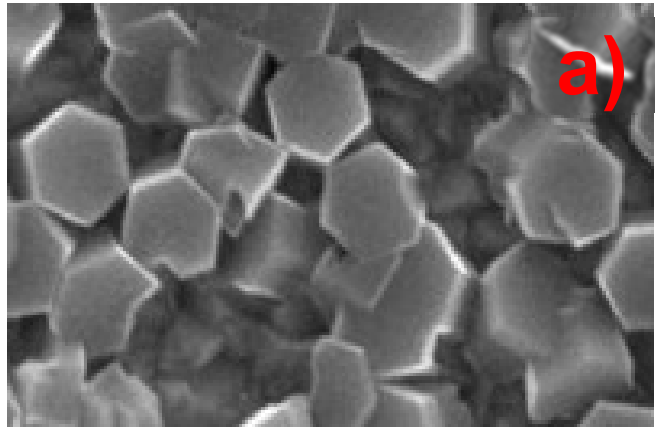

$200 \mathrm{~nm}$

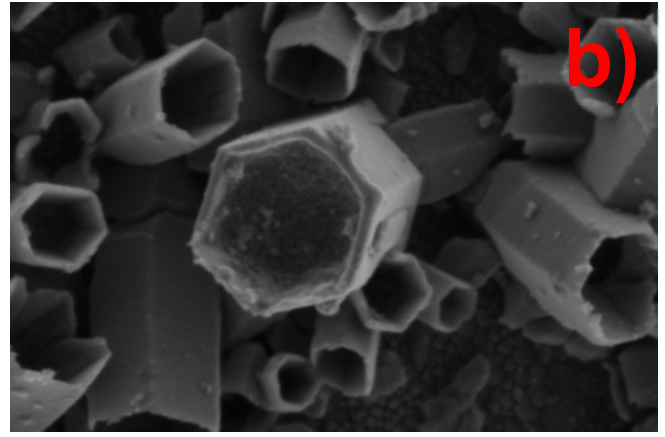

$200 \mathrm{~nm}$

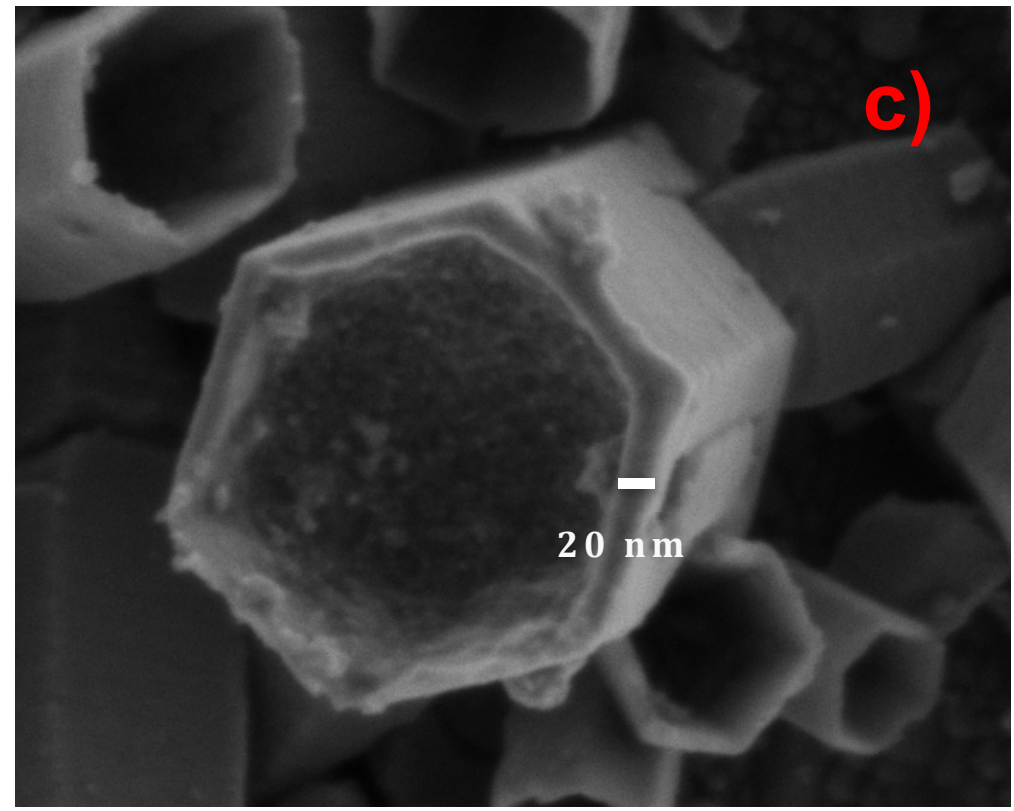

Figure 1. SEM images of electrodeposited $\mathrm{ZnO}$ nanostructures: a) As deposited $\mathrm{ZnO}$ nanocolumns, b) $\mathrm{ZnO}$ nanotubes after etching and regrowth, c) detail of nanotubes showing the thickness of the wall. 

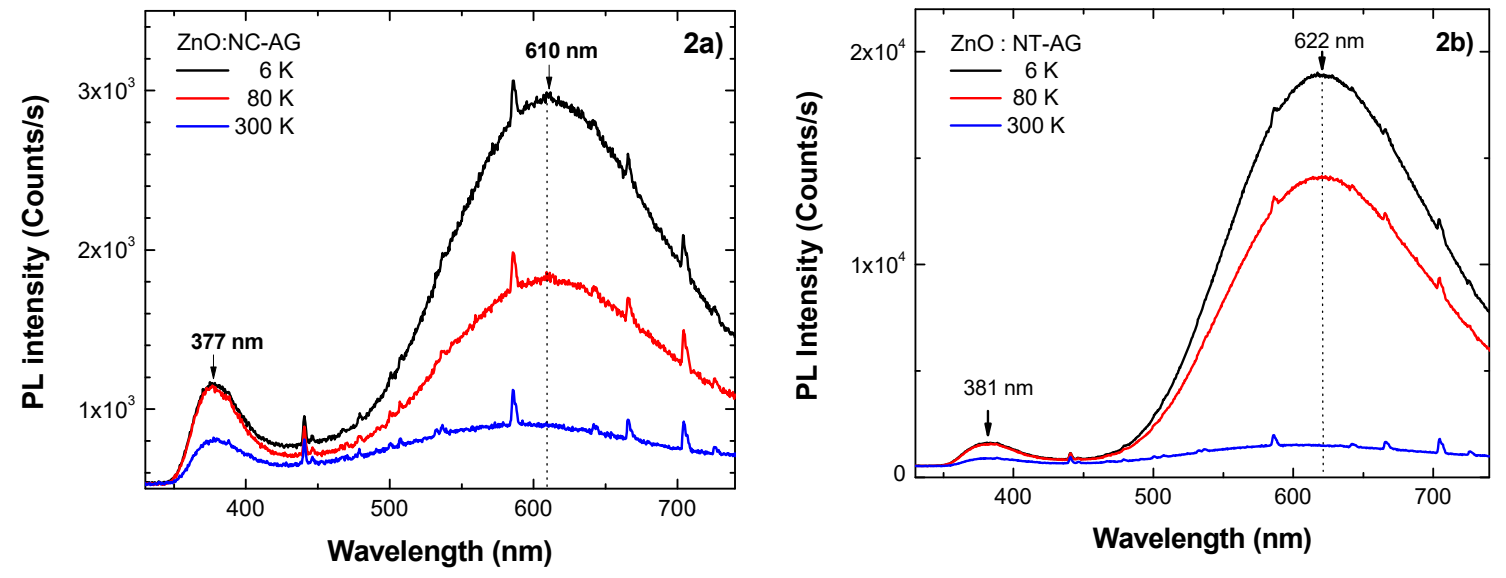

Figure 2. Photoluminescence spectra of as grown electrodeposited $\mathrm{ZnO}$ nanostructures: a) As deposited $\mathrm{ZnO}$ nanocolumns, b) $\mathrm{ZnO}$ nanotubes after etching and regrowth of $\mathrm{ZnO}$ nanocolumns.
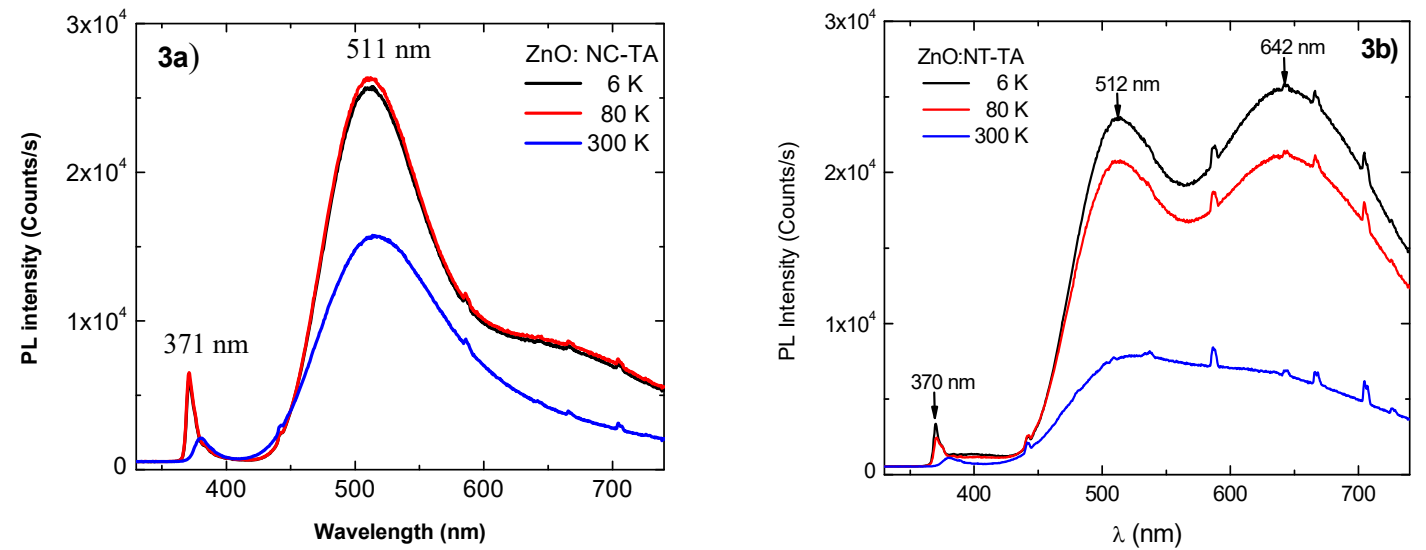

Figure 3. Photoluminescence spectra of electrodeposited $\mathrm{ZnO}$ nanostructures after thermal annealing: a) Nanocolumns (NC-TA), b) Nanotubes (NT-TA). 


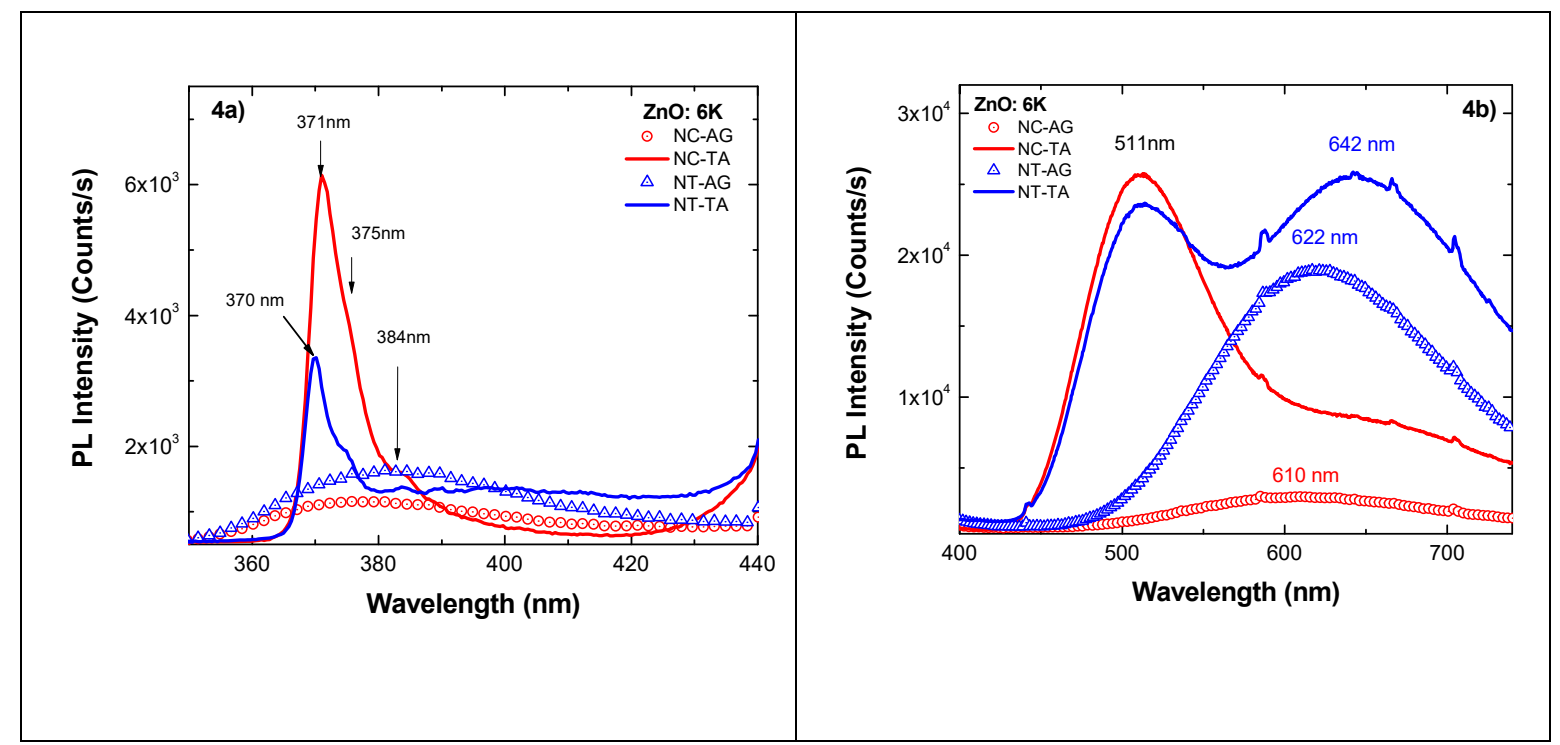

Figure 4. Comparison of photoluminescence spectra recorded at $6 \mathrm{~K}$ before and after annealing for $\mathrm{ZnO}$ nanostructures: a) Excitonic part (NBE region), b) Deep levels (DL region) 


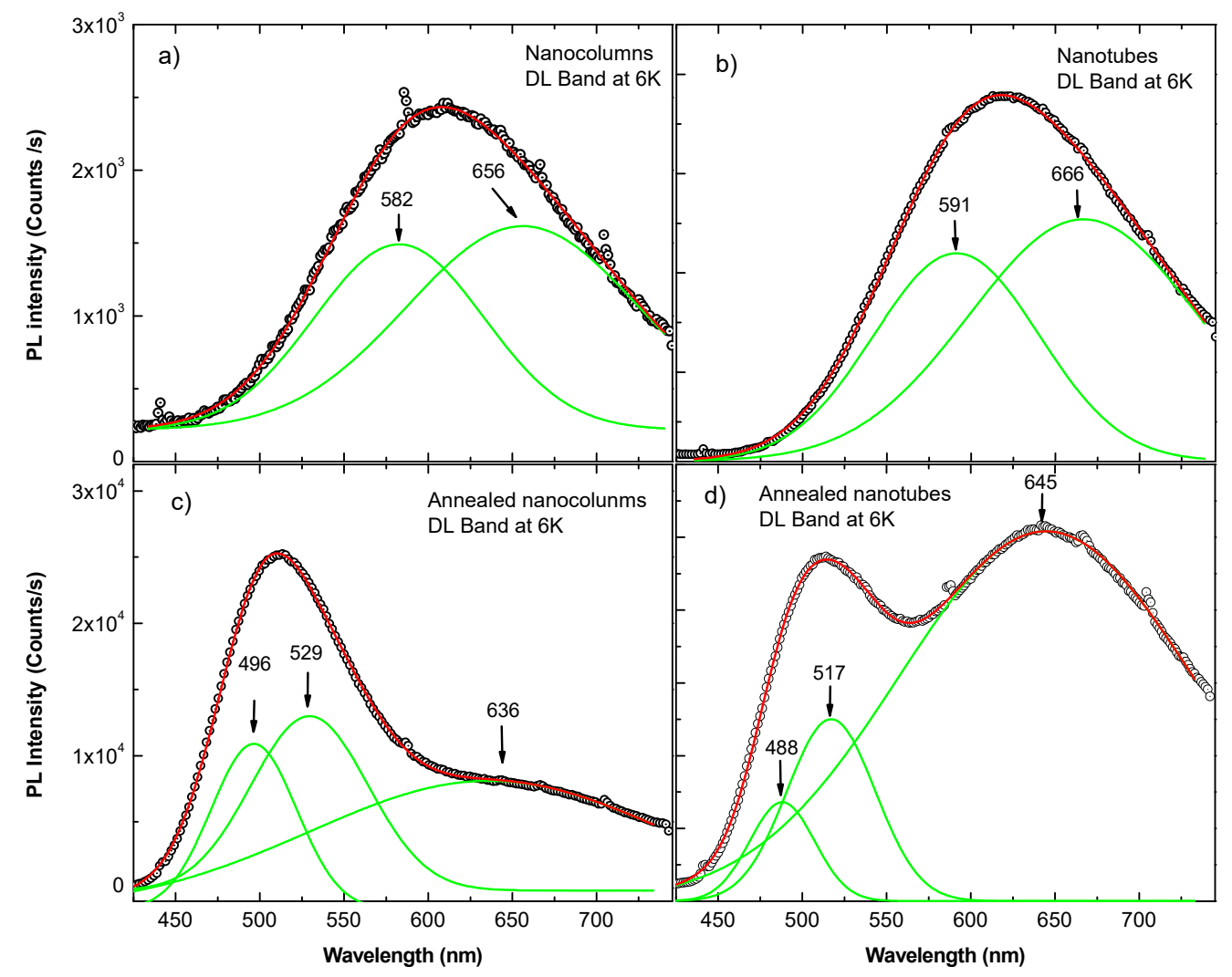

FIGURE 5. Fit of PL spectra in the DL region recorded at $6 \mathrm{~K}$ for all samples studied in this work. a) Nanocolumns, b) Nanotubes, c) annealed nanocolumns, d) annealed nanotubes. 


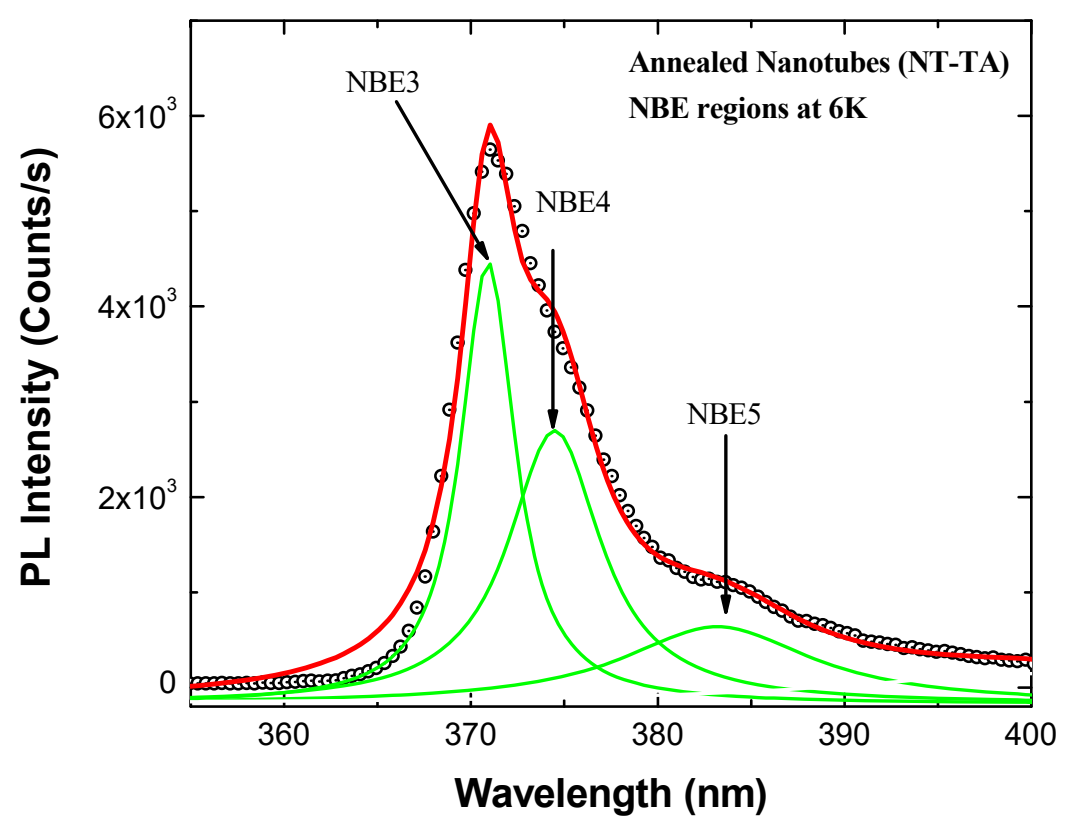

Figure 6. Fit of PL spectra in the NBE region recorded at $6 \mathrm{~K}$ for the annealed nanotubes. 


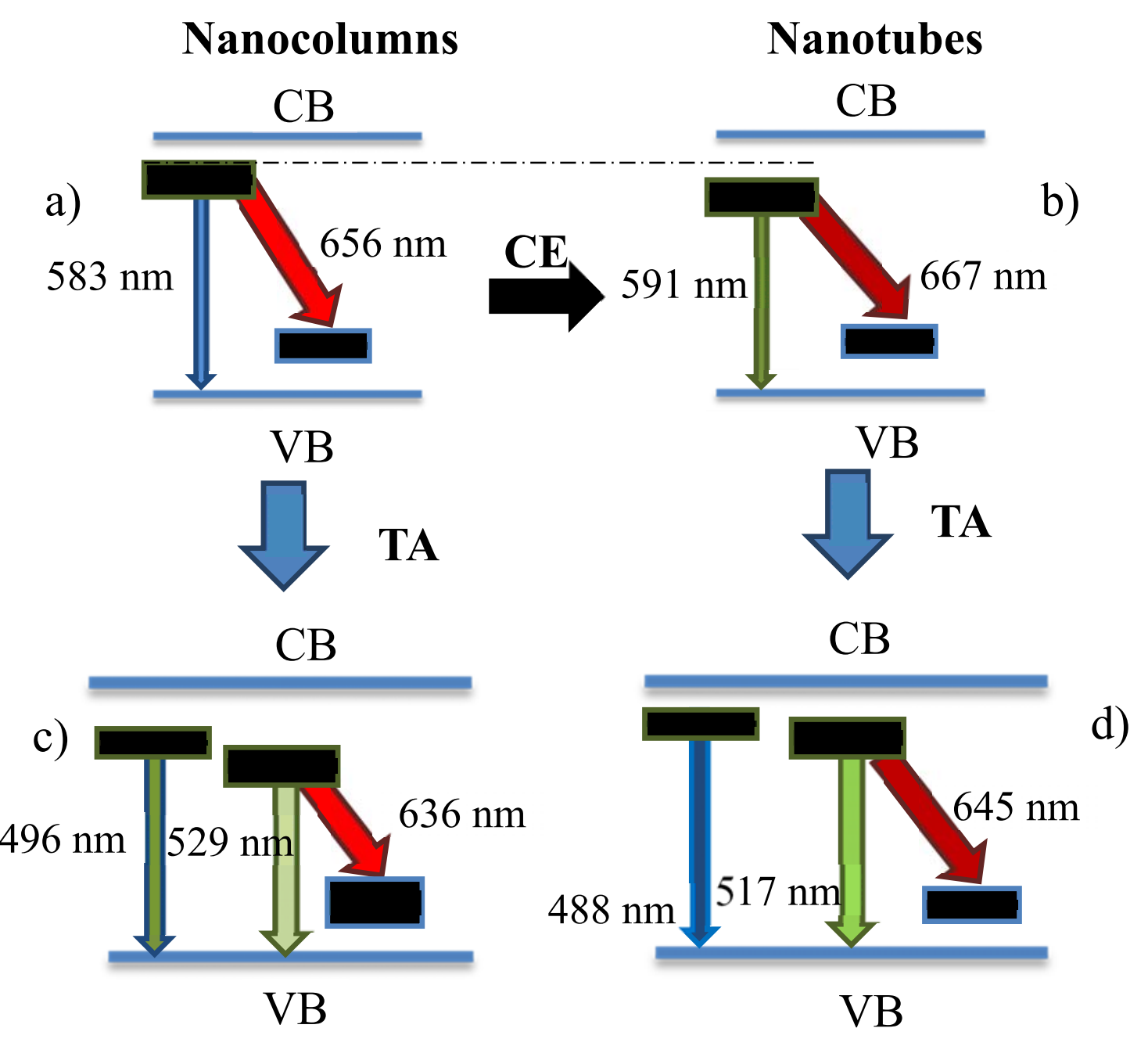

Figure 7. Band diagram schema to model the energy levels of involved defects in radiative transition in the DL region. CE and TA stand for Chemical Etching and Thermal Annealing, respectively. 